\title{
Effects of FR91 on immune cells from healthy individuals and patients with non-Hodgkin lymphoma
}

\author{
V. R. M. Lombardi ${ }^{1}$, E. Martinez ${ }^{2}$, R. Chacon $^{2}$, I. Etcheverria ${ }^{1}$ and R. Cacabelos ${ }^{1}$ \\ ${ }^{1}$ EBIOTEC, Cellular and Molecular Immunology Division, La Coruña, Spain and ${ }^{2}$ Georgian Alternative Medicine, \\ Madrid, Spain
}

Because of the importance of identifying cell immunophenotypes, considerable effort has gone into developing rapid identification methods for cell surface antigen involved in the modulation of immune system. The main objective of the present work was to evaluate the activity of FR91, a standardized lysate of microbial cells of the Bacillus genus, on peripheral blood lymphocytes from healthy individuals (HI) and patients with non-Hodgkin lymphoma (NHL) by following sequential changes in the immunophenotype of mixed-cell populations, as well as in the cytokine production.

Peripheral blood lymphocytes were obtained from $15 \mathrm{HI}$ and from $10 \mathrm{NHL}$ patients. $500 \mu \mathrm{l}$ of whole blood was diluted $1: 1$ with RPMI 1640 containing $10 \%$ foetal bovine serum, and FR91 was added at corresponding tubes at the concentration of 10,25 and $50 \mu 1 / \mathrm{ml}$, respectively, and incubated $24 \mathrm{~h}$ at $37^{\circ} \mathrm{C}$ in a humified $5 \% \mathrm{CO}_{2}$ atmosphere. After incubation, $100 \mu$ of blood was added to individual tubes and stained with anti-human CD3-FITC, anti-human CD25-FITC, anti-human CD69-FITC, anti-human HLADR-PE, anti-human CD4-PE and anti-human CD8-PE. After incubation and washing, tubes were passed on a flow cytometer and percentages of positive cells were calculated. For cytokine quantification, a multiplex bead array assays, which permit simultaneous cytometric quantitation of IL-1 $\beta$, IL-2, IL-6, IL-8, IL-10 p70, IL-12, IFN- $\gamma$, TNF- $\alpha$ in solution by capturing these to spectrally distinct beads, has been used.

The results of FR91 activity at the three different concentrations show a significant increase with respect to untreated controls of CD3 $(P=0.02)$ and $\mathrm{CD} 8(P=0.02)$ antigen expression in the HI group, while a higher increase in CD3 $(P=0.002)$ and CD4 $(P=0.002)$ was observed in the NHL group. A significant increase in IL-2 $(P=0.014)$, IL-6 $(P=0.001)$, IL-12 $(P=0.003)$, IFN- $\gamma(P<0.0001)$ and TNF$\alpha(P=0.002)$ cytokine expression was observed in the HI group. Similar results were observed in the NHL group: IL-1 $(P=0.03)$, IL-6 $(P=0.008)$, IL-12 $(P<0.0001)$, IFN- $\gamma(P<0.017)$ and TNF- $\alpha(P=0.012)$.

The significant increase of $\mathrm{CD}^{+}$lymphocytes in both studied groups suggests a possible use of FR91 in patients with altered function of the immune system. In addition, the increase in IL-2, IL-6, IL-12 and TNF- $\alpha$ cytokine production, can be one of the underlying mechanisms by which FR91 control cell proliferation. 